\title{
Sütsüz Dağı (Antalya) ve Çevresinin Endemik Bitkileri ve Tehlike Kategorileri
}

\author{
Hakkı DEMIRELMA*1, Yavuz BAĞCI ${ }^{2}$, Hüseyin DURAL ${ }^{1}$, Kuddisi ERTUĞRUL ${ }^{1}$ \\ ${ }^{1}$ Selçuk Üniversitesi Fen Fakültesi Biyoloji Bölümü, Konya, Türkiye \\ ${ }^{2}$ Selçuk Üniversitesi, Eczacılık Fakültesi, Temel Eczacılık Bilimleri, Farmasötik Botanik Ana Bilim \\ Dalı, Konya, Türkiye
}

Geliş / Received: 17/04/2018, Kabul / Accepted: 08/08/2018

\begin{abstract}
$\ddot{O} \mathbf{z}$
Bu araştırma 1999-2003 y1lları arasında ülkemizin önemli bitki alanlarından birisi olan Sütsüz Dağ1 ve çevresinde yayılış gösteren endemik bitkileri tespit etmek amacıyla yapılmıştır. Antalya il sınırları içerisinde yer alan araştırma alanının kuzey ucu Konya - Antalya il sınırını oluşturur. Araştırma alanı Davis'in kareleme sistemine göre C4 karesinde yer almaktadır. Araştırma sonucunda bölgeden 25 familya ve 60 cinse ait 92 takson tespit edilmiştir. Çalışma alanındaki endemik taksonlar IUCN kategori 3.1 versiyonuna göre değerlendirilmiştir. Değerlendirme sonucunda 1 taksonun (\% 1.09) CR (Çok tehlikede), 5 taksonun (\% 5.44) EN (Tehlikede), 10 taksonun (\% 10.87) VU (Zarar görebilir), 23 taksonun (\% 25.0) NT (Tehdit altına girebilir) ve 53 taksonun (\% 57.60) LC (En az endişe verici) kategorisinde yer aldığ 1 belirlenmiştir.
\end{abstract}

Anahtar Kelimeler: Endemik, IUCN, Sütsüz Dağı, Tehlike Kategorileri

\section{The Endemic Plants of Sütsüz Mountain (Antalya) and its Surroundings and Their Threat Categories}

\begin{abstract}
This study were carried out between 1999 and 2003 to determine the endemic plants of Sütsüz Mountain and its surrounding that is one of the most important plant area in Turkey. The study area is located in northern part of Antalya province and it forms aborder between Konya and Antalya provinces. According the Davis's Grid systems it placed in C4 square. According to the results of study, 25 families, 60 genera and 92 taxa were identified in study area. Endemic plants of study area were evaluated by IUCN Catagories Version3.1. According to results, the rates of IUCN categories as follows; CR (Critically endangered) 1 taxon (1.09\%), EN (Endangered) 5 taxa (5.44 \%), VU (Vulnerable) 10 taxa (10.87\%), NT (Near threatned) 23 taxa (25.0\%) and LC (Least corcern) 53 taxa $(57.60 \%)$ repectively.
\end{abstract}

Keywords: Endemic, IUCN, Sütsüz Mountain, Threat Categories

\section{Giriş}

Ülkemiz gerek endemik takson sayısı bakımından gerekse de tür çeşitliliği bakımından dünyada oldukça önemli bir yere sahip olup, tüm Avrupa ile kıyasladığında endemik takson ve tür sayısı bakımından hiçte azımsanmayacak sayıda bitki çeşitliliğine sahiptir. Türkiye Bitkileri Listesinde belirtildiği gibi 11707 taksondan 3649'u endemik olup endemizm oranı \% 31.82'dir (Güner vd., 2012). Son yıllarda özellikle Türk botanikçiler tarafından çok sayıda endemik takson bilim dünyasına tanıtılmış ve Türkiye Florası'na büyük oranda katkı sağlanmıştır. Son yıllarda araştırma bölgesi civarından bilim dünyası için çok sayıda yeni taksonlar tanımlanmıştır, bunlar: Aethionema alanyae H.Duman Duman (1994), Minuartia asiyeae H.Duman Duman (1998), Allium karacae Koyuncu Koyuncu (1994), Psephellus hadimensis (Wagenitz, Ertuğrul \& Dural) Wagenitz Wagenitz ve Hellwig (2000), Allium goekyigiti Ekim, H.Duman \& Güner Ekim vd. (1999), Pentanema alanyense H.Duman \& Anderb. Duman ve Anderberg (1999), Arabis alanyensis H.Duman Duman (2001), Chrysophthalmum gueneri Aytaç \& Anderb. 
Aytaç ve Anderberg (2001), Nonea dumanii Bilgili \& Selvi Bilgili vd. (2012), Arenaria mcneillii Aytaç \& H. Duman Aytaç ve Duman (2004), Origanum husnucan-baserii H. Duman, Aytaç \& A. Duran (Duman vd., 1995). Gerçekleştirilen bu çalışmayla, şimdiye kadar ayrıntılı olarak çalışılmamış olan Sütsüz Dağı ve çevresinde yayılış gösteren endemik taksonları tespit edilerek Türkiye Florası'na katkıda bulunulmuştur.

\section{Materyal ve Metot}

Araştırma bölgesi, Hadim (Konya) ilçesinin yaklaşık $60 \mathrm{~km}$ güneybatısında ve Konya iline yaklaşı $150 \mathrm{~km}$. uzaklıkta bulunmaktadır. Araştırma bölgesinde bulunan tek yerleşim merkezi Fakırçalı'dır. Ayrıca yörede Alanya'ya bağlı köylerin yaylaları bulunmaktadır. Gökbel yaylası, Cindi yaylası, Beyreli yaylası, Fakırçalı yaylası, Gömülgen yaylası, Eğerbel yaylası gibi. Bölgede oldukça sarp yükseltiler ile Gevne Çayı ve Arpalık Çayının oluşturduğu dar ve yüksek vadiler görülür. Araştırma bölgesinin büyük bir kısmını Kireçsiz Kahverengi Orman Toprakları kapsamaktadır. Kireçsiz Kahverengi oluşumu gösteren sert kalker eski olup Permiyen yaşlıdır. Araştırma alanının bir kısmında Kireçsiz Kahverengi Topraklar ve bazı yerlerde de çıplak kayalıklar bulunmaktadır (Anonim, 1974). Çalışma alanında paleozoyik çökellerini Karbonifer ve Permiyen yaşlı formasyonlar, Mesozoik çökellerini de Triyas ve Jura yaşı formasyonlar oluşturur. Karbonifer ve Permiyen çökelleri kuvars elemanlı kumtaşları ve resifal kireçtaşlarından oluşmuştur. Triyas ve Jura çökellerini ise kireçtaşları, dolomitik kireçtaşları, şeyl, kumtaşı ve çakıl taşları oluşturur. Çalışma alanında karbon çökelleri arasında bulunan kuvars arenitler, çoğunlukla sarıms1kahverengi ve bej bir görünüşe sahiptir. Kuşak Dağı tabanda Alt Permiyen yaşl1, Arpalık bölgesinin kuvars arenitleri üzerinde uyumlu olarak gelir. Üstten ise bol algli
Mizzia'lı kireçtaşlarını, Haydar formasyonuna ait Göztaşı üyesinin stromatolitik-oolitik kireçtaşları uyumlu olarak örter (Turan, 1985). Araştırma alanına en yakın istasyon olarak Hadim belirlenmiştir. Konya Meteoroloji Müdürlüğü'nden Hadim'e ait yağış ve sıcaklık ile ilgili iklimsel bilgilere göre Hadim'in yıllık yağgş ortalaması $664.5 \mathrm{~mm}$, yıllık sicaklık ortalamas $9.6{ }^{\circ} \mathrm{C}$ 'dir (Anonim, 1999). Yağış, yaz ve sonbahar mevsimlerinde azalmakta, kış ve ilkbahar mevsimlerinde ise artmaktadır. Buna göre yağış rejimi kış, ilkbahar, sonbahar ve yaz (KİSY) şeklinde sıralanmakta ve Doğu Akdeniz birinci tip yağış rejimi tipine girmektedir (Akman, 1990). Bölgenin iklimini karakterize edecek çeşitli özellikler De Martonne-Gottman, De Martonne ve Emberger formülleri ile ortaya çıkarılmıştır. De Martonne-Gottman metoduna göre; Hadim için elde edilen değer $\mathrm{I}=18$ 'dir. $\mathrm{Bu}$ değer Hadim için yarı-kurak, nemli iklimi gösterir (Akman, 1990). Emberger'in Yağış-Sıcaklık Emsali metoduna göre; $\mathrm{Q}=76$ değeri bulunur. İndis değerlerinden bakıldığ zaman Hadim az yağışlı Akdeniz iklimine girmektedir (Akman, 1990). Emberger'in Kurak Devre Formülüne göre; $\mathrm{S}=0.4$ olarak bulunur. $\mathrm{Bu}$ sayı 5'ten küçük olduğu için istasyon Akdeniz'lidir (Akman, 1990). Araştırma materyalini, 1999-2003 yılları arasında yapılan arazi çalışmaları sonucunda bölgeden toplanan bitki örnekleri oluşturmaktadır. Bitkilerin değişik vejetasyon devrelerine rastlayan Nisan-Eylül ayları arasında yapılan arazi çalışmaları sonucunda 1300 civarında bitki örneği toplanmıştır. Araziden toplanan bitkiler kurutulmuş, numaralandırılmış ve herbaryum örneği haline getirilmiştir. Bitki örnekleri Selçuk Üniversitesi Fen Fakültesi KNYA Herbaryumu'nda saklanmaktadir. Toplanan bitki örnekleri teşhis edilirken "Türkiye Floras1" adlı eserden faydalanılmıştır (Davis, 1965-1985; Davis vd., 1988; Güner vd., 2000). Bitki listesinde yer alan taksonların Latince isimleri ve yazar 
adları "Türkiye Bitki Listesi” eserine göre düzenlenmiştir.Endemik bitkilerin tehlike kategorileri "Türkiye Bitkileri Kırmızı Kitabı" adlı esere göre değerlendirilmiştir (Ekim vd., 2000). Çalışma alanındaki endemik bitki listesi verilirken, familyalar ve familyalar altındaki taksonlar alfabetik sira ile verilmiştir. Endemik bitki listesinde yer alan özellikle CR, EN, VU kategorilerinde yer alan taksonların tehlike durumlarının formüllerinin açılımı şöyledir: VU B1a: Yayılış alanı $20.000 \mathrm{~km}^{2}$, den az, ciddi derecede parçalanmış ya da sadece 10 veya daha az yerde bulunur. VU Bab(i): Yayılış alan1 $20.000 \mathrm{~km}^{2}$,den az, ciddi derecede parçalanmış veya sadece 10 ya da daha az yerde bulunur. Yayılış alanında düşüş var. VU B1ab(i,iii): Yayılış alanı 20.000 $\mathrm{km}^{2}$, den az, ciddi derecede parçalanmış ya da sadece 10 veya daha az yerde bulunur. Yayılış alanı, habitatın alanı, yayılış ve/veya niteliğinde düşüş var. EN B1ab(i): Yayılış alanı $5000 \mathrm{~km}^{2}$, den az, ciddi derecede parçalanmış ya da sadece 5 veya daha az yerde bulunur. Yayılış alanında düşüş var. EN B1ab(i,iii): Yayılış alanı $5000 \quad \mathrm{~km}^{2}$ 'den az, ciddi derecede parçalanmış ya da sadece 5 veya daha az yerde bulunur. Yayılış alanı, habitatın alanı, yayılışı ve/veya niteliğinde düşüş var. CR B1ab(i,iii): Yayılış alanı 100 $\mathrm{km}^{2}$, den az, ciddi derecede parçalanmış ya da sadece tek bir yerde bulunur. Yayılış alanı, habitatın alanı, yayılış ve/veya niteliğinde düşüş var (IUCN, 2001).

\section{Sonuç ve Tartışma}

Çalışma alanında yapılan floristik araştırmalar sonucunda 92 endemik takson tespit edilmiştir. Tespit edilen bu taksonlardan: $\mathrm{CR}$ (Çok tehlikede) kategorisinde 1 takson (\% 1.09), EN ( Tehlikede) kategorisinde 5 takson (\% 5.44), VU ( Zarar görebilir) kategorisinde 10 takson (\% 10.87), NT (Tehdit altına girebilir) kategorisinde 23 takson (\% 25.0) ve LC (En az endişe verici) kategorinde 53 takson (\% 57.60) tespit edilmiştir (Tablo 1).Çalışma alanımız yakınında yer alan Gevne Vadisi barındırdığı bitki türleri bakımından ülkemizde önemli bir yere sahiptir. $\mathrm{Bu}$ sebeple yalnızca Doğu ve Güneydoğu Anadolu Bölgelerinde yayılış gösteren Diplotaenia hayridumanii Pimenov \& Kljuykov, Opopanax persicus Boiss., Heracleum pastinacifolium C. Koch subsp. pastinacifolium ve Oenanthe sophiae Schischk. türlerinin bu bölgede yaşamlarını sürdürdügüüü ve bölgenin ilginç bir mikroklima özelliğine sahip olduğu bildirilmiştir (Duman vd., 2000). 1845 yılında Geyik Dağ Hedysarum anatolicum Amirahm. \& Kaz.Osaloo'un tanımlanmasından yaklaşık 150 yıl sonra bölgemizde yayılış gösterdiği tarafımızdan tespit edilmiştir (Ertuğrul vd., 2003). Ayrica monotipik cins olan Crenosciadium siifolium Boiss. \& Heldr. ve Leucocyclus formosus Boiss. subsp. amanicus (Rech fil) Hub.-Mor. \& Grierson'un alanda yayılış gösterdiği belirtilmiştir (Duman vd., 2000).

Tablo 1. Araştırma alanındaki endemik taksonların tehlike kategorilerine göre dağılımları ve oranları.

\begin{tabular}{ccc}
\hline $\begin{array}{c}\text { Tehlike } \\
\text { Kategorileri }\end{array}$ & Takson sayısı & $\begin{array}{c}\text { Yüzde (\%) } \\
\text { Oranı }\end{array}$ \\
\hline $\begin{array}{c}\text { CR (Çok } \\
\text { tehlikede) }\end{array}$ & 1 & 1.09 \\
\hline EN ( Tehlikede) & 5 & 5.44 \\
\hline $\begin{array}{c}\text { VU (Zarar } \\
\text { görebilir) }\end{array}$ & 10 & 10.87 \\
\hline $\begin{array}{c}\text { NT (Tehdit altına } \\
\text { girebilir) }\end{array}$ & 23 & 25.0 \\
\hline $\begin{array}{c}\text { LC (En az endişe } \\
\text { verici) }\end{array}$ & 53 & 57.60 \\
\hline
\end{tabular}

Endemik Taksonların IUCN Kırmızı Liste Kategorileri (2001) Sürüm 3.1 Listesi

AMARYLLIDACEAE

Allium goekyigitii Ekim, H.Duman \& Güner EN B1ab(i, iii)

Allium isauricum Hub.-Mor. \& Wendelbo VU B1a

APIACEAE

Ferulago aucheri Boiss. LC.

ASPARAGACEAE 
Asparagus coodei P.H.Davis EN B1ab(i, iii)

Bellevalia clusiana Gyriseb. LC.

Bellevalia tauri Feinbrun LC.

Hyacinthella glabrescens (Boiss.) K. Person \& Wendelbo NT.

Muscari anatolicum Cowley \& N. Özhatay. NT.

Muscari aucheri (Boiss.) Baker LC.

Muscari bourgaei Baker LC.

Muscari discolor Boiss. \& Hausskn. NT.

Muscari racemosum Mill. VU B1ab(i, iii)

Ornithogalum alpigenum Stapf. NT.

ASTERACEAE

Achillea teretifolia Willd. LC.

Centaurea deflexa Wagenitz VU B1ab(i)

Cicerbita variabilis (Bornm.) Bornm. LC.

Helichrysum arenarium (L.) Moench subsp. aucheri (Boiss.) Davis \& Kupicha LC.

Inula anatolica Boiss. LC.

Pentanema alanyense H.Duman \& Anderb.

CR B1ab(i, iii)

Senecio farfarifolius Boiss. \& Kotschy LC.

Tanacetum cadmeum (Boiss.) Heywood, subsp. cadmeum LC.

\section{BORAGINACEAE}

Alkanna sieheana Rech. f. NT.

Onosma isauricum Boiss. \& Heldr. LC.

Onosma sieheana Hayek VU B1ab(i)

Onosma stenoloba Hausskn. ex Riedl LC.

BRASSICACEAE

Aethionema alanyae H.Duman VU B1ab(i,

iii)

Arabis aubrietioides Boiss. LC.

Aubrieta canescens (Boiss.) Bornm. subsp. canescens LC.

Hesperis kotschyi Boiss. LC.

CAMPANULACEAE

Asyneuma compactum (Boiss. \& Heldr.)

Damboldt NT.

Asyneuma limonifolium (L.) Janch. subsp. pestalozzae (Boiss.) Damboldt LC.

Asyneuma linifolium (Boiss. \& Heldr.) Bornm. subsp. linifolium LC.

Campanula macrostyla Boiss. \& Heldr. NT.

Campanula pterocaula Hausskn. NT.

Campanula trachyphylla Schott \& Kotschy NT.

\section{CAPRIFOLIACEAE}

Pterocephalus pinardii Boiss. LC.

\section{CARYOPHYLLACEAE}

Arenaria acerosa Boiss. LC.

Arenaria tmolea Boiss. LC.

Bolanthus frankenioides (Boiss.) Bark. var. fasciculatus (Boiss. \& Heldr.) Bark. NT.

Dianthus cibrarius Clem. LC.

Dianthus recognitus Schischk. NT.

Gypsophila curvifolia Fenzl. LC.

Minuartia leucocephala (Boiss.) Mattf. LC.

Saponaria prostrata Willd. subsp. prostrata LC.

Silene capitellata Boiss. LC.

Silene caramanica Boiss. \& Heldr. LC.

\section{CONVOLVULACEAE}

Convolvulus galaticus Rostan ex Choisy LC.

\section{CRASSULACEAE}

Rosularia chrysantha (Boiss.) Tahkt. LC.

FABACEAE

Astragalus acmonotrichus Fenzl. LC.

Astragalus hirsutus Vahl. LC.

Astragalus lydius Boiss. LC.

GERANIACEAE

Erodium absinthoides Will. subsp. absinthoides LC.

Erodium cedrorum Schott \& Kotschy subsp. cedrorum NT.

Geranium glaberrimum Boiss. \& Heldr. NT. IRIDACEAE

Crocus biflorus Mill. subsp. isauricus (Siehe ex Bowles) B.Mathew LC.

Gladiolus anatolicus (Boiss.) Stapf LC.

\section{LAMIACEAE}

Ajuga bombycina Boiss. NT.

Lamium garganicum L. subsp. nepetifolium (Boiss.) R. Mill. LC.

Marrubium globosum Montbret \& Aucher ex Benth. subsp. micranthum (Boiss. \& Heldr.) P.H.Davis NT.

Nepeta isaurica Boiss. \& Heldr. LC.

Origanum saccatum P.H.Davis NT.

Phlomis armeniaca Willd. LC.

Phlomis monocephala P.H.Davis NT.

Phlomis russeliana (Sims) Benth. LC. 
Salvia adenocaulon P.H.Davis EN B1ab(i, iii)

Salvia heldreichiana Boiss. ex Benth. LC.

Salvia potentillifolia Boiss. \& Heldr. ex Benth. NT.

Scutellaria orientalis L. subsp. pectinata (Montbret \& Aucher ex Benth.) J.R.Edm.

LC.

Scutellaria salviifolia Benth. LC.

Sideritis bilgeriana P.H.Davis VU B1ab(i)

Sideritis brevibracteata P.H.Davis VU B1ab(i, iii)

Sideritis libanotica Labill. subsp. violascens (P.H.Davis) P.H.Davis NT.

Stachys citrina Boiss. \& Heldr. subsp. citrina NT.

Stachys cretica L. subsp. anatolica Rech. LC.

Stachys iberica Bieb. subsp. iberica var. densiplosa R. Bhattachajee LC.

Thymbra sintenisii Bornm. \& Aznav. subsp. isaurica P.H.Davis NT.

\section{LILIACEAE}

Fritillaria acmopetala Boiss. subsp. wendelboi Rix EN B1ab(i)

\section{PAPAVERACEAE}

Papaver apokrinomenon Fedde LC.

\section{PINACEAE}

Abies cilicica (Ant. \& Kotschy) Carr. subsp. isaurica Coode \& Cullen LC.

\section{PLANTAGINACEAE}

Linaria corifolia Desf. LC.

Linaria genistifolia (L.) Mill. subsp. confertiflora (Boiss.) P.H.Davis LC.

Linaria genistifolia (L.) Mill. subsp. polyclada (Fenzl) P.H.Davis NT.

Veronica dichrus Schott \& Kotschy NT.

Veronica multifida L. LC.

RANUNCULACEAE

Consolida glandulosa (Boiss. \& Huet)

Bornm. LC.

Consolida raveyi (Boiss.) Schröd. LC.

\section{ROSACEAE}

Amelanchier parviflora Boiss. var. dendata Browicz LC.

SAPINDACEAE
Acer hyrcanum Fisch. \& Mey. subsp. sphaerocaryum Yalt. VU B1a

\section{SCROPHULARIACEAE}

Scrophularia scopolii Hoppe ex Pers. var. longirostrata Heywood EN B1ab(i, iii)

VIOLACEAE

Viola isaurica Contondr. \& Quézel VU B1ab(i, iii)

\section{XANTHORRHOEACEAE}

Asphodeline prismatocarpa J. Gay ex Boiss.

VU B1ab(i, iii)

Asphodeline rigidifolia (Boiss.) Baker LC

\section{Teşekkür}

$\mathrm{Bu}$ çalışmayı, 98/114 nolu proje ile destekleyen BAP (Bilimsel Araştırmalar Projeleri Koordinatörlüğüne) teşekkür ederiz.

\section{Kaynaklar}

Akman, Y. 1990. İklim ve Biyoiklim. Palma Yayın ve Dağıtım, 319 s. Ankara.

Anonim, 1974. Doğu Akdeniz Havzası Toprakları Serisi, Toprak Su Genel Müdürlüğü-Ankara.

Anonim, 1999. Meteoroloji Bülteni, Meteoroloji Bölge Müdürlüğü, Konya.

Aytaç, Z. \& Anderberg, A.A. 2001. A new species of Chrysophtalmum Schultz Bip. (Asteraceae-Inula) from Turkey. Botanical Journal of the Linnean Society, 137, 211-214.

Aytaç, Z., Duman, H. 2004. Six new taxa (Caryophyllaceae) from Turkey. Annales Botanici Fennici, 41, 213-221.

Bilgili, B., Coppi, A., Selvi, F. 2012. Nonea dumanii sp. nov. (Boraginaceae) from the Taurus mountains (South Turkey). Nordic Journal of Botany, 30, 546-542.

Davis, P.H. 1965-1985. Flora of Turkey and the East Aegean Island. Vol. 1-9, Edinburgh University., Press, Edinburgh.

Davis, P.H., Mill, R.R., Tan, K. 1988. Flora of Turkey and the East Aegean Island (Supplement 1), Vol. 10. Edinburgh University., Press, Edinburgh. 
Duman, H. 1994. A new species from SouthAnatolia- Aethionema alanyae (Brassicaceae). The Karaca Arboretum Magazine, 2(4), 171-176.

Duman, H., Aytaç, Z., Ekici, M., Karavelioğulları, F.A, Dönmez, A., Duran, A. 1995. Three new species (Labiatae) from Turkey. Flora Mediterranea, 5, p:226.

Duman, H. 1998. A new Species of Minuartia (Caryophyllaceae) from South Anatolia. The Karaca Arboretum Magazine, 4,59-162.

Duman, H., Anderberg, A.A. 1999. An undescribed species of Pentanema Cass. (Asteraceae-Inuleae) from Turkey, with notes on the phylogenetic status of the genus. Botanical Journal of the Linnean Society, 129, 333-338.

Duman, H., Aytaç, Z., Karevelioğulları, F. 2000. Gevne Vadisi'nin (HadimAlanya) Florası. Kırsal Çevre ve Ormancılık Sorunları Araştırma Derneği Yayın, No: 9. Ankara.

Duman, H. 2001. A new species of Arabis L. (Brassicaceae) from South Anatolia. Botanical Journal of the Linnean Society, 137, 87-90.

Ekim, T., Duman, H., Güner, A. 1999. A new Allium L. Species from Anatolia: Allium goekyigitii Ekim, H. Duman \& Güner. Karaca Arboretum Magazine, 5,27-34.

Ekim, T., Koyuncu, M., Vural, M., Duman, H., Aytaç, Z., Adigüzel, N. 2000. Red Data Book of Turkish Plants (Pteridophyta and Spermatophyta). Türkiye Tabiatını Koruma Derneği, Yayın No: 18. Ankara.

Ertuğrul, K., Dural, H., Bağc1, Y. 2003. "The Rediscovery of Sartoria Boiss. \& Heldr. (Pișiktaşağı, Leguminosae/Fabaceae): A Monotypic Endenic Genus of Turkey. The Karaca Arboretum Magazine, 7(1), 13-18.

Güner, A., Özhatay, N., Ekim, T., Başer, K. H.C. 2000. Flora of Turkey and the East Aegean Island (Supplement 2), 11. Edinburgh University., Press, Edinburgh.
Güner, A., Aslan, S., Ekim, T., Vural, M., Babaç, M. T. (Edlr.) 2012. Türkiye Bitkileri Listesi (Damarlı bitkiler). Nezahat Gökyiğit Botanik Bahçesi ve Flora Araştırmaları Derneği Yayını. İstanbul.

IUCN Red List Categories: Version 3.1. 2001. Prepared by the IUCN Species Survial Commission. IUCN, Gland Switzerland and Cambridge, UK.

Koyuncu, M. 1994. A new species of Allium L. (Liliaceae) from Southern Anatolia. The Karaca Arboreteum Magazine, 2 (4), 177-180.

Turan, A. 1985. Beyreli (Hadim-Konya) Dolayının Jeoloji İncelemesi, Yüksek Lisans Tezi. Selçuk Üniversitesi Fen Bilimleri Enstitüsü, Konya.

Wagenitz, G., Hellwig, F.H. 2000. The genus Psephellus Cass. (Compositae, Cardueae) revisited with a broadened concept Free Access. Willdenowia, 30,34 . 